\title{
On regular and random two-dimensional packing of crosses
}

\author{
Ralf Stannarius $^{1} \mathbb{D}$. Jonas Schulze ${ }^{1}$
}

Received: 28 June 2021 / Accepted: 10 November 2021 / Published online: 15 December 2021

(c) The Author(s) 2021

\begin{abstract}
Packing problems, even of objects with regular geometries, are in general non-trivial. For few special shapes, the features of crystalline as well as random, irregular two-dimensional (2D) packing structures are known. The packing of 2D crosses does not yet belong to the category of solved problems. We demonstrate in experiments with crosses of different aspect ratios (arm width to length) which packing fractions are actually achieved by random packing, and we compare them to densest regular packing structures. We determine local correlations of the orientations and positions after ensembles of randomly placed crosses were compacted in the plane until they jam. Short-range orientational order is found over 2 to 3 cross lengths. Similarly, correlations in the spatial distributions of neighbors extend over 2 to 3 crosses. There is no simple relation between the geometries of the crosses and the peaks in the spatial correlation functions, but some features of the orientational correlations can be traced to typical local configurations.
\end{abstract}

Keywords Packing fraction $\cdot$ Crosses $\cdot$ Jamming $\cdot$ Orientational order $\cdot$ Positional correlations $\cdot$ Experiment

\section{Introduction}

Prediction of packing configurations of identical objects is in general a tough problem, even for very simple particle geometries and without spatial restrictions. Such problems have a long history. For example, the densest regular threedimensional (3D) packing of spheres was already conjectured by Johannes Kepler more than four centuries ago. Nevertheless, it took until the beginning of 21 st century to find the proof for this conjecture [1]. Spatial restrictions like an enclosure in cylindrical, cuboid or other containers add even more complexity [2-6].

Besides spheres, other particle shapes as for example ellipsoids [7], polyhedra [8-12] or spherocylinders [13] have attracted the interest of researchers. Studies on packing problems in 3D included also non-convex particles like stars or hexapods [14-16]. The practical relevance of the latter type of objects may be found in construction industry and architecture [17, 18], when one is looking for a material that forms stable aggregates of a desired shape, but on the other hand can be disassembled again with little efforts

Ralf Stannarius

ralf.stannarius@ovgu.de

1 Institute of Physics, Otto von Guericke University, Magdeburg, Germany for a structural redesign. An excellent and comprehensive review of 3D packing problems of regular particles of various shapes has been published by Torquato and Stillinger [19].

Often, when a set of randomly placed regular objects is compacted, a regular crystalline ground state is not reached. Instead, more or less random local arrangements are obtained both in experiments and simulations [20]. For example, pouring hard objects into a container does not lead to the spontaneous formation of a regular, crystalline configuration. Even shaking or shearing ensembles of spheres leaves them in arrangements where packing is random, and the packing fraction of around $\phi_{\text {rcp }} \approx 64 \%$ remains well below the optimum value for the crystalline configurations [21-26]. This jammed state is irregular and in general much less densely packed than the optimum space-filling arrangement, not only for spherical particles.

In two dimensions (2D), packing problems are often simpler than in 3D, but nevertheless far from being generally understood. A proof of the densest (hexagonal) disk packing was found by Fejes [27]. Since the hexagonal lattice optimizes 2D packing locally and globally, filling a plane with disks yields an almost crystalline structure with few defects. These defects will gradually disappear when the system is appropriately agitated. For more complex shapes like polygons [28, 29], ellipses and dimers [30], irregular 
packing structures were reported at the onset of jamming. Some non-convex shapes composed of straight or bent segments have been considered in numerical simulations e. g. in Refs. [31-33]. In contrast to the number of numerical studies, experimental work has been performed only scarcely.

Altogether, we have to distinguish strictly three different packing problems: The first one is the question of optimal packing, in an infinitely extended volume or under particular geometrical constraints (e. g. [3, 5, 6, 11, 32, 34, 35]). For convex centrosymmetric particles, densest packing structures are crystalline [34]. In general, however, this state is not reached after compaction of a random ensemble of particles.

The second problem is the description of externally agitated matter, for example granular ensembles under mechanical vibrations (e. g. [36-42]). Such a system in a non-perfectly ordered state is able to explore the configuration space. Here, the entropy of the individual configurations comes into play. In simulations, one can force the system by repeated growth and shrinking of the ensemble [43], or by adding Brownian motion [44-47]. In many respects, these systems can be considered as analogues of colloidal crystals. Container vibrations play the role of thermal fluctuations. The individual particles can find some more compact configurations. In some of these systems, local order may develop spontaneously. For vibrated rods in quasi-2D monolayers, for example, a tetratic local orientational order was discovered in experiments [40]. Tetratic order was also reported in simulations of ensembles of densely packed rectangles [43], Similar investigations were performed for other shapes such as kite-shaped [47], bow-shaped [45], or square [41, 44] particles.

The third problem is the understanding of the jammed state which is reached in an athermal system when the packing fraction is gradually increased by compaction. When the jamming transition has occurred, the system is in a mechanical equilibrium. No further dynamics is considered. One important parameter is the space filling $\phi$ (area filling in 2D) at the jamming point. In the present study, we will focus on this third problem. We investigate cross-shaped objects with different aspect ratios of their arms, and determine the packing densities and structures that characterizes the onset of jamming.

The packing of cross-shaped objects in a plane has been analysed before in different contexts and under different aspects (e. g. [31-34]), since it is both interesting from a fundamental point of view and for practical purposes. We refer here to regular flat objects with fourfold symmetry, a horizontal and four vertical mirror planes $\left(D_{4 h}\right)$. Their extension in the third dimension is disregarded and we consider only their 2D projections. The two types of crosses considered in this study are shown in Fig. 1. The most relevant geometrical feature of crosses is their arm width to arm length
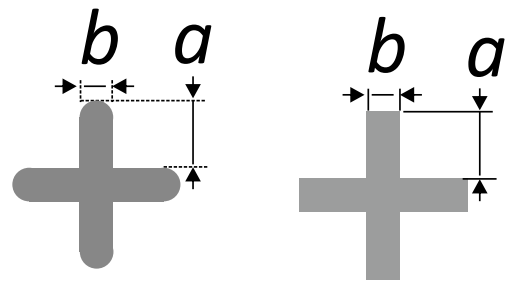

Fig. 1 Definitions of the arm length $a$ and width $b$ for rounded and simple crosses

ratio, which will determine their optimal packing structure (the one with the best area filling). We denote here the length of the arm as $a$, its width as $b$ (see Fig. 1), the aspect ratio as $\rho=b / a$, and the cross 'size' as $\ell=2 a+b$ (the distance of the ends of opposing arms). The simplest crosses have arms with flat ends. The limiting cases of those structures for $a \rightarrow 0, \rho \rightarrow \infty$ are squares and for $b \rightarrow 0, \rho \rightarrow 0$ crossed line segments. A slight variation is the cross shape with rounded arms.

There has been only little attention to experiments with crosses in 2D so far. The most relevant experimental study in the context of the present work was performed by Zheng et al. [31]. The authors conducted shear experiments with stress-birefringent objects. The major goals of that study were the characterization of the onset of jamming, the distribution of stresses in the ensemble, and the pressure characteristics near the jamming point. The objects are nearly equivalent to crosses with rounded ends with $\rho \approx 0.4$ in our notation, except for rounded corners in the center. Zheng et al. employed uniaxial and biaxial compression. Both approaches led to slightly different jamming points: under uniaxial compression, the jamming transition was reached at about $47.5 \%$ space filling ( $\phi=0.475)$, while under biaxial compression this value was slightly higher, on average by about $0.25 \%$. A systematic investigation of the random 2D packing characteristics of such objects has not yet been performed there.

Similar crosses with rounded ends of their arms were also treated in some theoretical studies $[32,33]$. This particular shape detail facilitates numerical simulations. Marschall and Teitel [33] studied the shearing of crosses with twofold and fourfold symmetries in a numerical simulation. In our notation, their cross shapes correspond to an aspect ratio of $\rho=0.5$. Focus of that paper was on the relation between pressure and strain rate in shear experiments, but the authors also reported a value of the packing fraction. They found the jamming transition at $\phi=0.66$. The authors also reported numerical results for pair correlation functions, and they computed the tetratic correlation, separately for neighboring crosses in the direction along the arms and diagonal to them.

The purpose of this study is an experimental generation of random packings of crosses with different aspect 
ratios, and the statistical characterization of the achieved packings in terms of mean global packing fraction, spatial correlations between the particle positions and orientations in the jammed state, and the influence of boundary effects. The present manuscript is organized as follows: Before we describe the experiments with random arrangements of the particles, we will shortly recollect structures that are best fitted candidates for an optimum space filling. This allows us to judge the packing efficiency reached by compaction in comparison to the maximum achievable $\phi$ for a given cross shape. Within the limits of $0<\rho<\infty$, very different optimum crystalline arrangements exist [34]. While our uniaxial compression technique naturally does not result in crystalline order, some features of these structures will be found locally in the random arrangements that we study experimentally.

In the main part of this study, we present experiments with crosses which are arranged randomly on a table and then compacted to a jammed cluster that cannot be compressed further without deforming or breaking the objects. The packing achieved that way represents a good approximation for random close packing of the respective cross geometry. These irregular arrangements have packing fractions $\phi$ that are considerably smaller than those of the optimum regular structures, particularly when the aspect ratio $\rho$ is low (thin crosses). We will discuss the short-range spatial correlations in these arrangements and the mutual orientational correlations of neighbors on the basis of experimental findings.

\section{Crystalline packings}

Before we introduce our experimental results, we recollect the regular crystalline arrangements found to be the best space-filling lattices. These configurations have been introduced by Atkinson and collaborators and published as Supplemental Material of Ref. [34]. Atkinson's study considers crosses with straight edges. These optimum packing structures for flat-ended crosses (Fig. 1 right) are shown in Fig. 2.

In sequence of increasing aspect ratio $\rho$, the densest crystalline packings are the tilted square TSQ (we name it after the geometrical shape of the unit cell marked in Fig. 2 by dashed lines), the rhombic end-to-end (ETE) and side-by-side (SBS) lattices named after the mutual positions of arms of neighboring crosses, and the tilted rhomboid (TRH). In Appendix A, we give details of the packing fractions that correspond to these configurations, and for the transitions between the best packing structures in dependence of the aspect ratio.

The complete graph of the fill fractions corresponding to the regular structures defined above is shown in Fig. 3. The dashed lines show the fill fractions of the different configurations where they are not the optimum structures. The

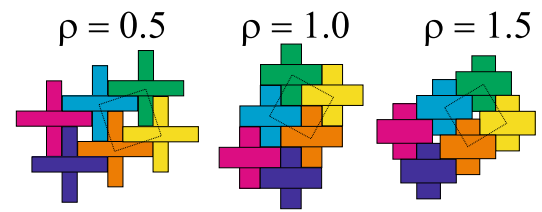

$\mathrm{TSQ}$, tilted square

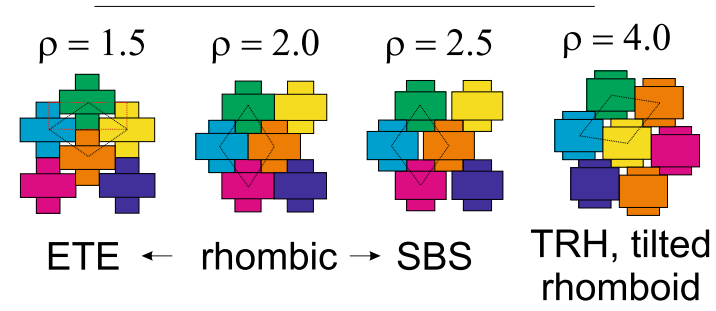

Fig. 2 Regular packings of crosses for selected aspect ratios $\rho=b / a$. Tilted square lattices are shown in the top row, rhombic and square lattices in the bottom row

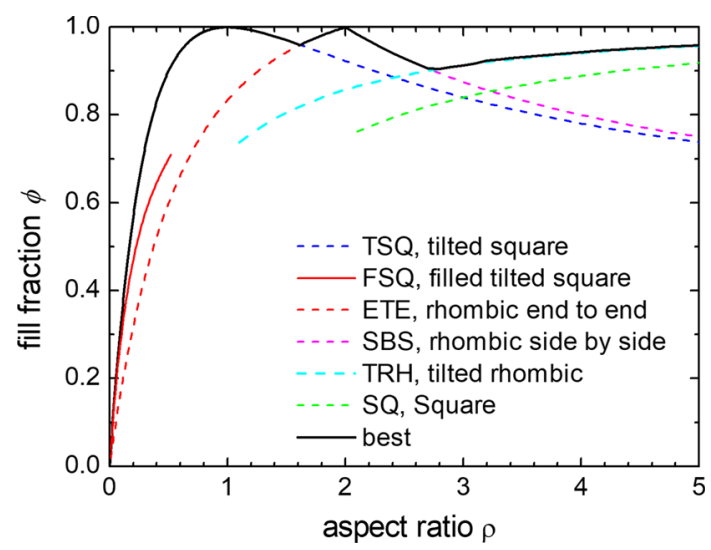

Fig. 3 Area filling fractions $\phi$ of several regular (crystalline) packings of crosses with different aspect ratios $\rho$. The black curve shows the densest packed structure found. The individual packing fractions exist in the following ranges: TSQ and SQ for all $\rho$, ETE for $\rho<2$, SBS for $\rho>2$, FSQ exists up to $\rho \approx 0.52$

solid black line is the plot of the optimum configurations for given $\rho$. While for $\rho \rightarrow 0$, the packing fraction reaches zero, a complete coverage of the $2 \mathrm{D}$ plane is reached when the arm width-to-length ratio is $\rho=1$. For broader arms, the packing becomes less efficient again before it reaches full coverage again when $\rho=2$. At $\rho \rightarrow \infty(a=0)$, the crosses degenerate to squares, and the optimum area filling reaches $\phi=1$ for the third time.

We mention in passing that all the above crystalline lattices have tetratic order with an orientational order parameter (see Sect. 3.4) of $q_{4}=1$. Several other regular configurations with more than one cross per unit cell are shown in Appendix B. None of them reaches a higher packing fraction than the optimum configurations described above. In our experiments we 
will also deal with crosses that have rounded ends, which are characterized by slightly different optimum packing structures.

Even though there is great confidence that the most efficient packing structures have been identified [34], there is no strict mathematical proof that denser configurations are excluded. The purpose of introducing these lower limits of densest packing here was to set a scale for the comparison with the experimental results on randomly packed crosses. These are described in the following sections.

\section{Random packings}

\subsection{Samples and experimental setup}

We use plastic crosses that are commercially available as spacers for tiling. They can be obtained in different aspect ratios. In the present study, we selected crosses for nominal 2 $\mathrm{mm}, 3 \mathrm{~mm}, 4 \mathrm{~mm}, 5 \mathrm{~mm}, 8 \mathrm{~mm}$ and $10 \mathrm{~mm}$ arm widths, corresponding to aspect ratios between 0.25 and 0.89 . In addition, crosses with an aspect ratio of 2 were $3 \mathrm{D}$ printed in the lab. We refer to the latter shortly as black crosses. Table 1 lists the geometrical dimensions of these five species.

The height of the crosses is between $3 \mathrm{~mm}$ for the smallest type and $6 \mathrm{~mm}$ for the largest. A static friction coefficient $\mu=0.3 \pm 0.05$ among the crosses has been estimated by measuring the tilt angle of a plane on which a cross starts to slide down from the surface of a second cross. The sliding friction coefficient of the crosses on the bottom plate is also around 0.3 .

The crosses are initially placed at random on a horizontal plate with three fixed rails that form three sides of a rectangular base (Fig. 4). We leave enough space between the crosses so that the initial area filling fraction is at least 2 to 3 times lower than in the jammed state, and we try to prepare a rather uniform distribution. Each experiment starts with a new initial configuration. After placing the crosses, this area is covered by a transparent glass plate. At the fourth side of the rectangular area, a slider can be manually moved in to shift the crosses and to compact the ensemble. The slider is first moved to a position where the crosses resist the advancement and a jammed state is reached. Then, a photo is taken. The slider is then pushed further with a force of a few Newton, which further leads to a slight compaction of the crosses configuration. A second photo is taken and the slider is then hit hard several times, in order to cause further compaction. Then, we reach a state where further hitting the moveable side bar does not lead to an improvement of the fill fraction, and a third photo is taken. The jamming point is assumed to be in the range between the first and third slider positions. Strictly, the first point where the slider experiences resistance should be defined as the jamming point of our system, but since this first contact is often established by only one or very few crosses, such a definition would slightly underestimate the behavior of an infinitely large system.

Image analysis is used to determine the positions and orientations of all crosses. From these data, we can not only determine the packing fractions, but also the spatial correlations between cross centers and their mutual orientations. The experiments were performed with sets containing between 166 and 500 crosses. The number of crosses and the number of repetitions of each experiment are listed in Table 2 in Appendix D.

\subsection{Area filling fraction}

The crosses are placed on a plane in a loose packing and then compressed laterally by a moving boundary as described above. Thereby, the optimum space filling structure is never reached, not even approximately. One of the reasons for that

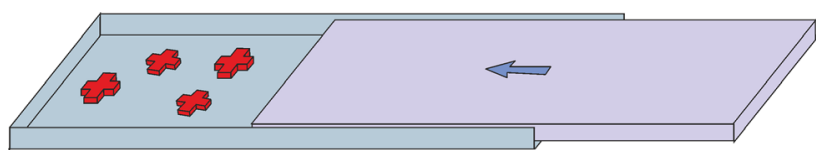

Fig. 4 Schematic drawing of the experimental setup with the table and slider
Table 1 Geometrical parameters for the plastic crosses used in our experiments

\begin{tabular}{lllllll}
\hline Nominal & $\rho$ & $a[\mathrm{~mm}]$ & $b[\mathrm{~mm}]$ & $\ell[\mathrm{mm}]$ & $r_{0}[\mathrm{~mm}]$ & Rounded \\
\hline $3 \mathrm{~mm}$ & 0.248 & 12.1 & 3.0 & 27.2 & 15.4 & No \\
$2 \mathrm{~mm}$ & 0.284 & 7.4 & 2.1 & 16.9 & 9.73 & Yes \\
$4 \mathrm{~mm}$ & 0.445 & 9.2 & 4.1 & 22.5 & 13.9 & Yes \\
$5 \mathrm{~mm}$ & 0.516 & 9.5 & 4.9 & 23.9 & 15.2 & Yes \\
$8 \mathrm{~mm}$ & 0.728 & 10.5 & 7.65 & 28.6 & 19.7 & No \\
$10 \mathrm{~mm}$ & 0.894 & 10.8 & 9.65 & 31.2 & 22.6 & No \\
Black & 2.0 & 5.0 & 10.0 & 20.0 & 18.0 & No \\
\hline
\end{tabular}

The lengths are accurate within $\pm 0.1 \mathrm{~mm}$, thus we round the aspect ratios to two digits in the following plots. The $2 \mathrm{~mm}, 4 \mathrm{~mm}$ and $5 \mathrm{~mm}$ crosses have arms with semicircular ends, the others are flat-ended. The arm length $a$ of the rounded crosses includes the rounded end. The minimal possible distance between centers of neighbors is $r_{0}$ 
is the non-convex geometry of the particles that facilitates catched states of the individual crosses. Small clips of typical photos of the experiment are shown in Figs. 5 and 6. We have chosen regions near the moveable slider. One notices that in Fig. 5, the thin crosses in contact with the slider are preferentially aligned diagonal to the edge, touching it with two arms, but this induced order does not penetrate much more than one cross diameter into the plane. This issue is discussed in more detail later. The two top images in Fig. 5 show the same area in two compaction steps. The left one shows the crosses after they first block the slider motion. The right one presents the same experiment after the slider was pushed hard several times towards the crosses, until no further rearrangement could be noticed. There is surprisingly little difference between the statistical properties of both configurations, a feature that is discussed next.

Figure 7 shows the result of 100 independent experiments (300 images) with $8 \mathrm{~mm}$ crosses $(\rho=0.73)$. We present three graphs, where the open symbols show the packing fraction $\phi$ after the first compaction phase, the filled symbols the packing fraction after the system is repeatedly hit from the side until no rearrangements occur any more. The packing fraction or area fill fraction $\phi$ is calculated by the area covered by crosses divided by the total area. The averages

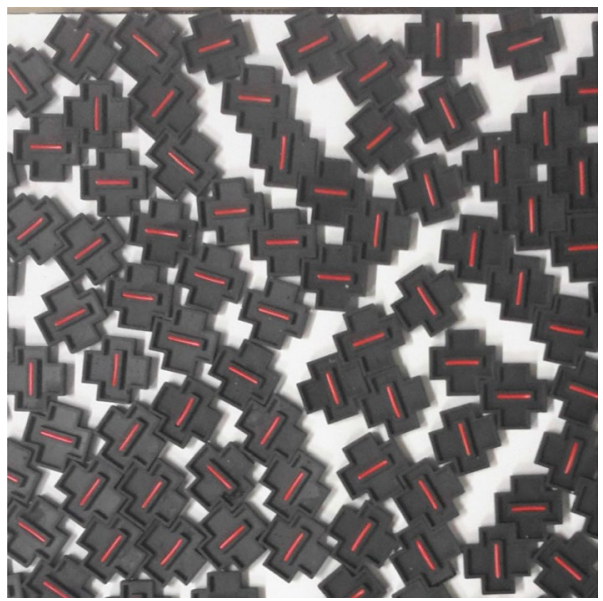

Fig. 6 Photo of jammed crosses with aspect ratio $\rho=2$. The positions and orientations of the crosses are identified by means of the red marker bars. The edge of the slider bar is seen at the top

of these measurements differ by less than 5\%. Figure 8 compares the packing fractions of random close packing (jamming point) of crosses of different aspect ratios with the curve of the optimum regular packing, TSQ for $\rho<1$. Two trends are evident in the figure: First, the random packing
Fig. 5 Photographs of randomly packed crosses. Top left: 3 $\mathrm{mm}$ crosses after first phase, loosely packed; Top right: $3 \mathrm{~mm}$ crosses after final compaction; Bottom left: jammed $5 \mathrm{~mm}$ crosses (arms with rounded ends); Bottom right: jammed 8 $\mathrm{mm}$ crosses. All images show a small $20 \mathrm{~cm} \times 20 \mathrm{~cm}$ clip next to the moving edge (top). Red labels merely serve as identifiers to facilitate image processing

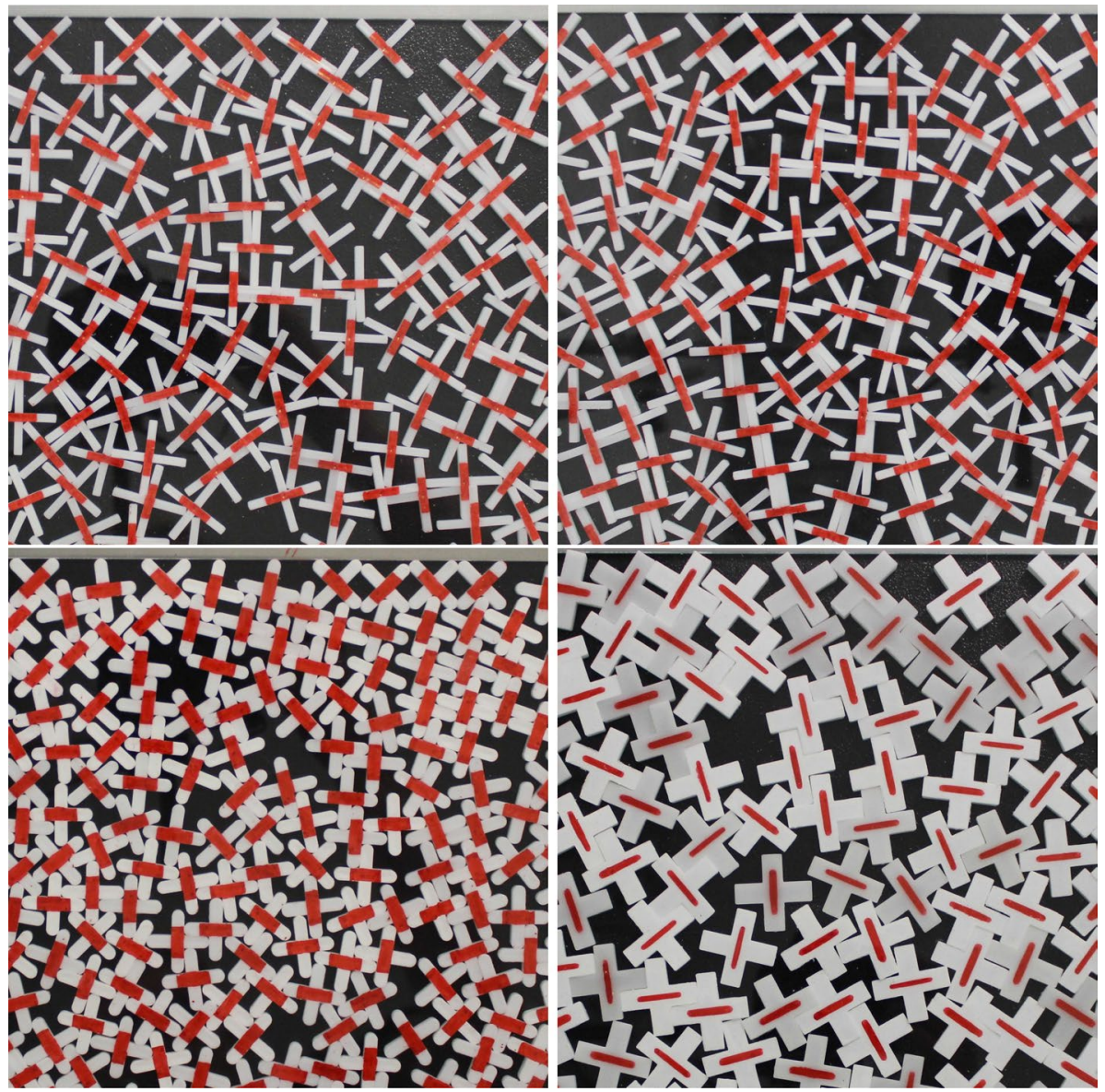




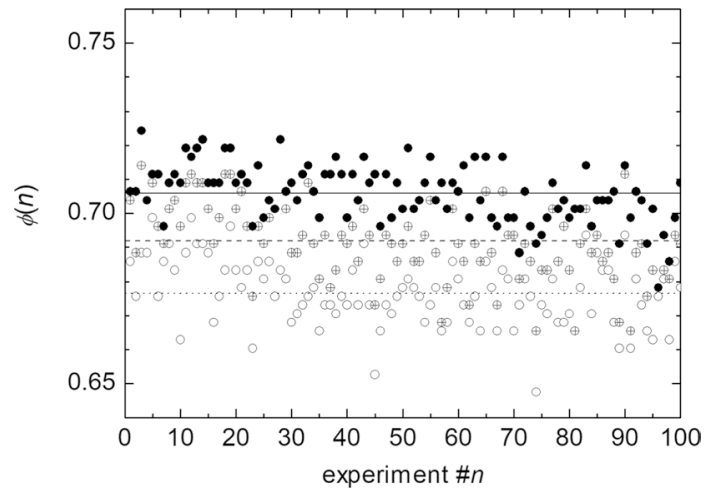

Fig. 7 The plot shows the packing fractions $\phi$ determined in 100 independent experiments (crosses with $\rho=0.73$ ). The symbols correspond to the value measured when the first blockage of the movable bar is reached (o), the value when the sample is further compactified with larger force $(\oplus)$, and the compaction reached after hard pushes of the bar $(\bullet)$. The corresponding averages are plotted as dotted, dashed and solid lines, respectively

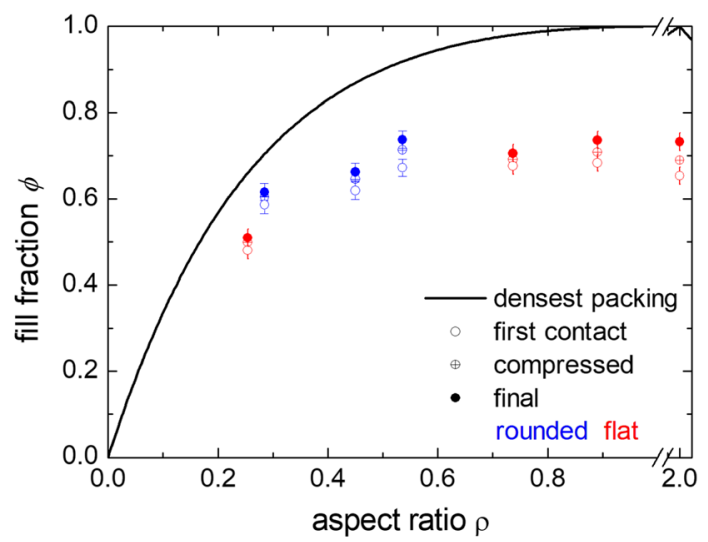

Fig. 8 Area filling fractions $\phi$ of jammed configurations of randomly packed crosses

fractions are much smaller than the regular optimum packing fractions for given aspect ratios (roughly $70 \%$ of the latter). The fill fraction $\phi(\rho)$ increases with increasing aspect ratio. The second trend is that crosses with rounded ends $(2 \mathrm{~mm}$, $4 \mathrm{~mm}$ and $5 \mathrm{~mm}$ crosses in our study) apparently achieve a somewhat larger fill fraction than one would expect by interpolation of the data of flat-ended crosses. This is intuitively clear: When crosses with flat ends touch each other with edges, further rotations are blocked unless the distance between the cross centers is increased again. With semicircular ends, the crosses can rotate with respect to each other to find denser configurations.

\subsection{Spatial correlations}

Irrespective of the overall random arrangement of the crosses, one finds a strong correlation of orientations and positions of neighbors. This is a consequence of the nonconvex character of the particles. Figure 9 shows the spatial correlation functions $\phi_{\mathrm{loc}}(r)$, which are proportional to the probabilities of finding crosses in a distance $r$ (center-center) from a given cross. In contrast to the numerical simulations of Marschall and Teitel [33], we averaged over all orientations of the connection vector $\mathbf{r}$. For an angular-dependent experimental characterization of these distributions, much more runs of the experiments would be needed in order to obtain a reliable statistics.

The spatial distances in these graphs have been normalized with the minimum

$r_{0}(\rho)=\sqrt{\left[(a+b)^{2}+b^{2}\right]}=\sqrt{\left[(1+\rho)^{2}+\rho^{2}\right]} \cdot a$,

defining the center-center distance of two crosses laying with their arms side by side (like those at the sides of the TSQ unit cell, Fig. 2). For a clearer presentation, we have multiplied the number densities $N(r)$ of crosses with the area $A_{0}$ of the single cross to obtain $\phi_{\text {loc }}$. Thus for large $r$, where the cross positions are uncorrelated, the mean area fill fractions $\phi$ shown in Fig. 8 are reached asymptotically, $\lim _{r \rightarrow \infty} \phi_{\text {loc }}(r)=\phi$

In addition to the dominant maxima near $r=r_{0}$ in each graph, one identifies several more peaks in the individual graphs. Their positions depend on the aspect ratios. Some of them are found in the vicinity of $2 r_{0}$, and all

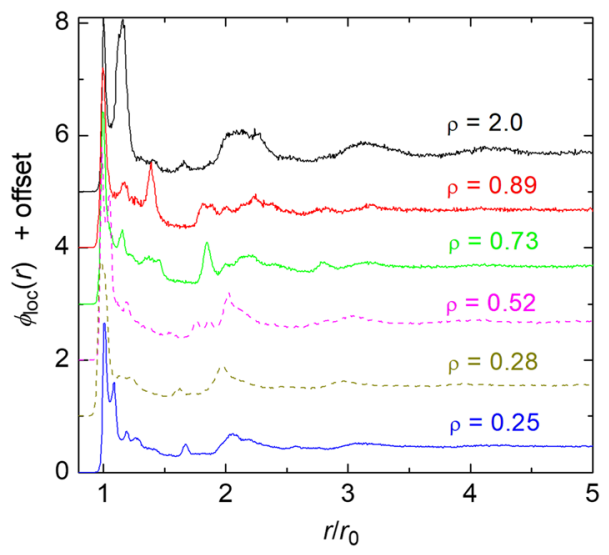

Fig. 9 Experimental results for the correlation of densities in dependence on the aspect ratio. The effective local fill fraction $\phi_{\text {loc }}$ is the product of the average number $N(r)$ of cross centers per area in a distance $r$ from a central cross, multiplied with the cross area $A_{0}$, and $r_{0}$ is the minimal possible distance of neighbors. In order to separate the graphs, we added offsets of $0,1,2, \ldots 5$ from bottom to top. Selected individual graphs are shown in Figs. 10 and 11 together with a peak assignment 
$\phi_{\mathrm{loc}}(r)$ graphs have a pronounced dip in the region around $1.5 r_{0}<r<2.0 r_{0}$, these distances are clearly underrepresented. The cross positions show noticeable correlations over distances of about 3 to $4 r_{0}$, i. e. about two cross diameters, particularly for the crosses with broader arms (larger $\rho)$. Since there is no obvious relation between the aspect ratios $\rho$ and the relative peak positions at first glance, their explanation needs a deeper analysis. We solved this problem at least partly, by identifying pairs of crosses in the images that are separated by distances near the peak positions. This reveals that some of the peaks, viz. $r_{1}$ to $r_{6}$ in Figs. 10a and b, are representative for typical pair configurations. Figure $10 \mathrm{c}$ sketches some of these configurations, where the green and purple crosses are separated by $r_{i}$. With light grey we marked intermediate crosses that stabilize the mutual orientations of the outer two.

\section{(a)}

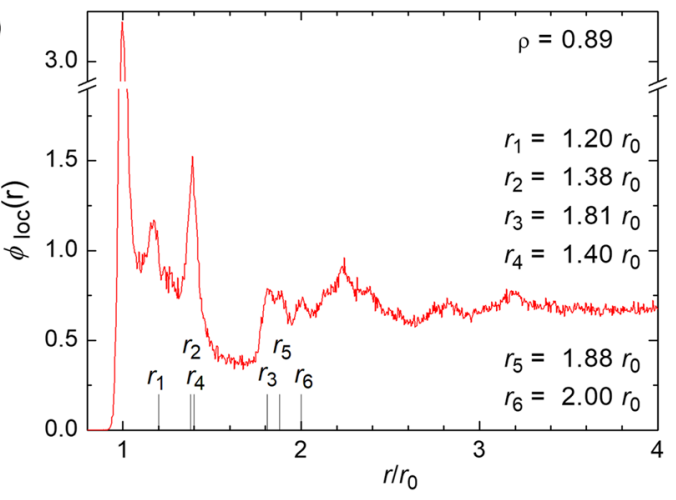

(b)

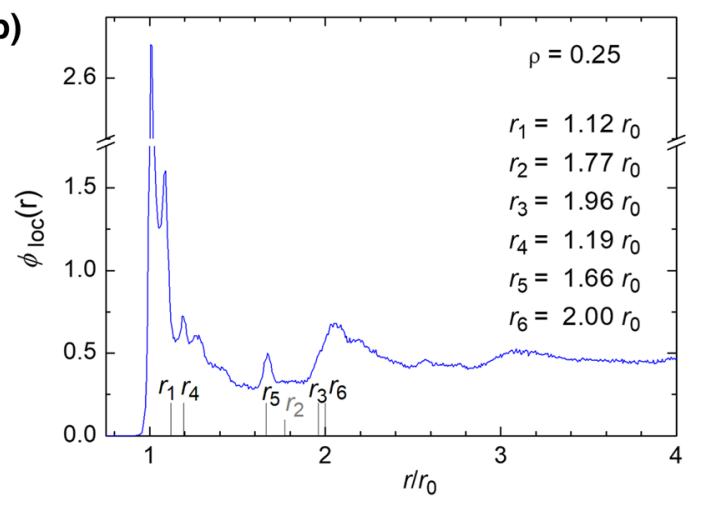

(c)

$$
\begin{aligned}
& r_{1}=a+\left(1+2^{-1 / 2}\right) b \\
& r_{2}=2 a+b \\
& r_{3}=2 a+2 b
\end{aligned}
$$

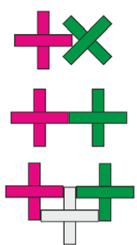

$$
\begin{aligned}
r_{4} & =\left[(a+2 b)^{2}+b^{2}\right]^{1 / 2} \\
r_{5} & =2^{1 / 2}(a+2 b) \\
r_{6} & =2 r_{0} \\
& =2\left[(a+b)^{2}+b^{2}\right]^{1 / 2}
\end{aligned}
$$
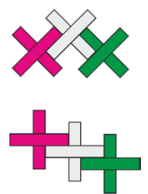

Fig. 10 Graphs $\mathbf{a}$ and $\mathbf{b}$ are identical with those in Fig. 9. c Shows the mutual positions and orientations of pairs that contribute dominantly to the corresponding peaks
Peaks near these six $r_{i}$ are primarily caused by a preference of the sketched local arrangements. The peak $r_{2}$ caused by end-to-end contacts is practically absent in the case of thin crosses, as seen in Fig. 10b. Note that all ratios $r_{i} / r_{0}$ depend on $\rho$. Therefore, peaks of similarly correlated pairs have different relative positions.

Figure 11 shows the spatial correlations of crosses with $\rho=2$ (3D-printed black crosses). Two essential new features are seen: First, a very pronounced peak appears next to the first maximum $r=r_{0}$. It is identified as the separation of crosses in end-to-end configuration, $r_{2}$. When they are perfectly aligned, the peak appears at $\approx 1.1 r_{0}$ for $\rho=2$. When the crosses are shifted sidewards respective to each other, the distance is larger, therefore this peak is rather broad. The second obvious feature is the pronounced appearance of peaks of the second and third coordination shells which are much less pronounced for crosses with $\rho<1$.

\subsection{Orientational correlations}

When discussing orientational order, the first question that needs to be clarified is whether the uniaxial compaction method, pushing one side bar, has an influence on the global ordering of the crosses. Is the configuration statistically isotropic, or is there an induced uniaxial order? Zheng et al. [31] have compared the uniaxial compression method with the results obtained by biaxial compression, and they have found slight differences between the two jammed states. The jamming point is slightly shifted to larger packing fractions for biaxial compression (see above), but during cyclic compression, the results of the two compression methods seem to converge. The biaxial compression is referred to as

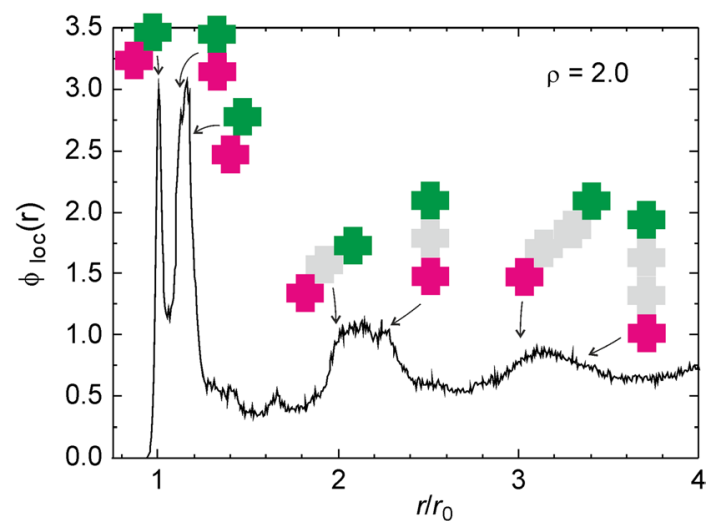

Fig. 11 Graphs of the spatial correlation of cross centers for the aspect ratio $\rho=2$. Note the pronounced differences to the previous samples. The minimum distance is $r_{0}=18 \mathrm{~mm}$. The large peak starting near $20 \mathrm{~mm} \approx 1.1 r_{0}$ is generated by crosses stacked end-to end. The configurations mainly responsible for the peaks are indicated by inset images. Note that there are well-pronounced peaks of the second and third coordination spheres 
'isotropic' in that paper, indicating that the authors assume a configuration where all spatial directions are equivalent. Yet it was not explicitly studied whether uniaxial compression has an impact on the isotropic ordering of the ensemble or whether it creates a 'memory' of the compression history with a preferential axis.

Our Fig. 5 indicates that at least at the borders, surface induced alignment is present, but we tried to find a quantitative measure for the anisotropy of the orientation of crosses in the central part. For such a quantitative description, we use the global tetratic bulk order parameter

$q_{4}=\left\langle\cos 4 \alpha_{i}\right\rangle$

where the $\alpha_{i}$ are the angles of the individual crosses respective to the sides of the rectangular box. The coordinate system is chosen such that this angle is zero when the arms are along or perpendicular to the sides formed by the confining rails. This choice is justified by symmetry arguments. We confirmed it by evaluating the more general quantity $\left\langle\cos 4\left(\alpha_{i}-\alpha_{0}\right)\right\rangle$ as a function of $\alpha_{0}$, which was found to yield its extremum near $\alpha_{0}=0$ for all cross types.

When computing $q_{4}$, we average over all crosses except those in the first layer near the rails. More accurately, we exclude all crosses whose centers are closer than one cross size to any of the side walls. We further average over all images for a given cross type. The result is that independent of the cross geometries, and independent of the state of compaction, all global order parameters $q_{4}$ of thin crosses $(\rho<1)$ were very small but systematically negative. Those crosses are somewhat more likely to be diagonal to the borders than parallel to them. The same is reflected in a prevalence of this orientation in the boundary layers (see, e. g. Fig. 5, top). The averages of $q_{4}$ over all experiments were of the order of -0.01 for all shapes of thin crosses. This means that the compaction method used here (uniaxial compression) had negligible influence on the global orientational order. The configurations can be assumed to be isotropic in reasonable approximation. The wide crosses with $\rho=2$ showed an equally weak orientational order, but with a positive sign of the global order parameter $q_{4}$. Here, the average was $q_{4}=0.014$. This value is still tolerably small to consider the system practically as isotropic, but the sign change shows that the broad crosses were somewhat more likely to be aligned with their arms parallel or perpendicular to the sliding bar rather than diagonal. This slight prevalence is induced by our preparation method of the jammed states. A simple explanation of this mechanism is given in Appendix C. From the arguments given there it is plausible to assume that similar effects will be found under biaxial compression, because the order induced at the fixed and moving bars is comparable.

Irrespective of the global isotropy, neighboring crosses show substantial correlations of their orientations. This is reflected in the plots in Fig. 12. Two of these graphs are shown

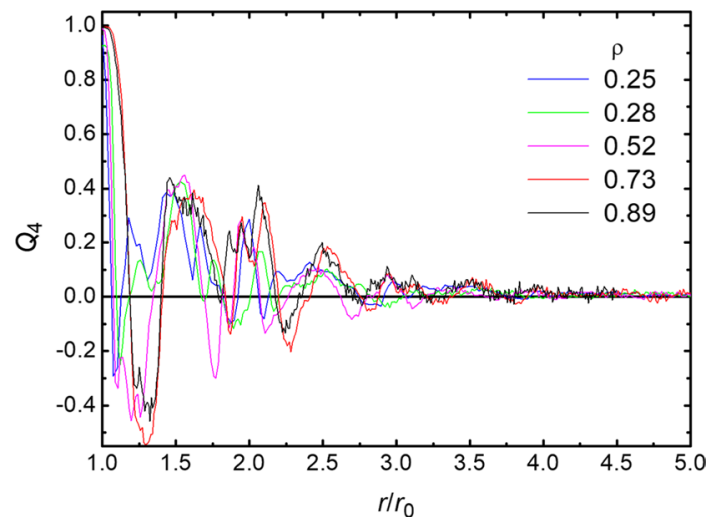

Fig. 12 Local order parameter $Q_{4}(r)$ of the surrounding crosses in distance $r$ from a central cross, averaged over all central crosses
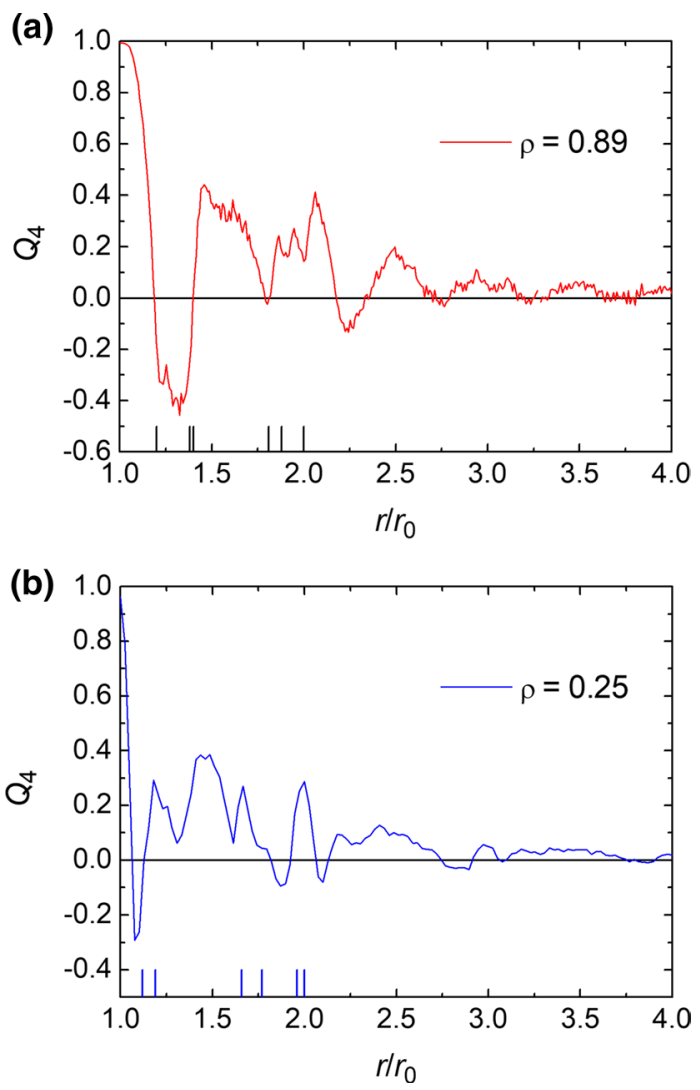

Fig. 13 Local orientational correlations parameter $Q_{4}(r)$ of the surrounding crosses in distance $r$ from a central cross for a $\rho=0.89$ and $\mathbf{b} \rho=0.25$. Markers indicate the labeled peak positions of $\phi_{\mathrm{loc}}$ in Figs. 10a,b

separately in Fig. 13. Here, we computed the local orientational correlation parameter

$Q_{4}(r)=\left\langle\cos 4 \varphi_{i}\right\rangle$ 
Here, we average over all crosses in distance $r$ from the central cross and $\varphi_{i}$ are the angles of the crosses $i$ in distance $r$ from the central cross with respect to the orientation $\alpha_{c}$ of the latter: $\varphi_{i}=\alpha_{i}-\alpha_{c}$. Because of the fourfold symmetry of the crosses, these angles are taken in a $90^{\circ}$ range. This order parameter $Q_{4}$ reaches its maximum +1 when two crosses are oriented with their arms parallel, and the minimum -1 indicates that the second cross is diagonal to the first one. With 150 to 300 experiments for each $\rho$ and between 150 and 250 crosses in each image, the statistics are based on data of at least $10^{6}$ pairs.

It is evident that for distances close to $r_{0}$, only aligned crosses can exist. Thus $Q_{4}\left(r_{0}\right)=1$ for all types of crosses. Then, the local order parameter drops to negative values because crosses in distances near $r_{1}$ preferentially contribute to a negative value of the $Q_{4}$. This orientational order is less perfect than that of the directly attached crosses, thus the negative peak reaches only values around -0.5 (for the thin crosses). The orientational correlation $Q_{4}(r)$ changes its sign with increasing $r$ several times. It finally vanishes over a distance of about $4 r_{0}$,

The large undulations of $Q_{4}(r)$ with $r$ are systematic, they represent characteristic features of the random packing structures. However, there appears to be no clear correlation between the peak positions in $\phi_{\mathrm{loc}}$ and those in the local orientational order, except the global maximum $Q_{4}\left(r_{0}\right) \approx 1$ and the local minimum $Q_{4}\left(r_{1}\right)$. Again, the wide crosses $(\rho=2)$ shown in Fig. 14 are an exception. There, the negative peak is suppressed, and the parameter $Q_{4}(r)$ is predominantly positive almost everywhere. The probability that the crosses in a certain distance are diagonal to the central cross is much lower than for the thin crosses. A pronounced positive peak at $r=2 r_{0}$ indicates aligned crosses in the 2 nd coordination sphere of the central cross.

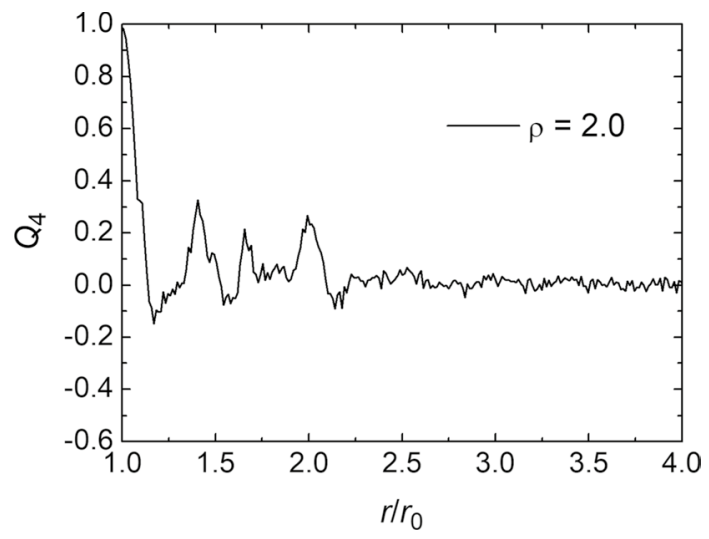

Fig. 14 Local orientational correlations parameter $Q 4(r)$ of the crosses in distance $r$ from a central cross for $\rho=2$

\section{Discussion and summary}

A few literature results obtained in numerical simulations can be used as benchmarks for our experimental results. Concerning the densest crystalline packing, our predictions for $\rho<1$ can be compared with results reported by Meng et al. [32] for rounded crosses. Their parameter $W$ is related to $\rho$ by $W=2 / \rho$. Densest packing reported in that paper reaches from about $\approx 0.36$ for $\rho=0.1$ (our TSQ value: 0.336 ) over $\approx 0.55$ at $\rho=0.2$ (our TSQ: 0.567 ) to about 0.92 for $\rho=0.8$ (our TSQ: 0.989). For thin crosses, these analytical results agree very well. Naturally, the differences between the densest packing of rounded and flat crosses increase with increasing arm width-to-length ratio $\rho$. Therefore, our structure of flat crosses reaches $\phi_{\max }=1$ when $\rho=1$, while the rounded crosses reach a smaller fill level at $W=2$. They leave open areas next to the rounded ends, while the crystalline packings of the flat end crosses can cover the complete area.

The result of our experiments on random packing are the following: The uniaxial compression creates an almost isotropic jammed state of crosses, with an induced orientational order restricted essentially to the first layer at the boundaries. The measurements of the slider positions and the related areas occupied by a fixed number of crosses provide the packing fractions at the jamming transition. These jamming transitions occur at fill fractions $\phi(\rho)$ much smaller than the optimum crystalline packing for all cross geometries studied. It turns out that the geometry of the arm ends (rounded or flat) has only little influence on the random packing efficiency. Even though one would intuitively assume that rounded ends lead to a denser packing, the effect is only in the range of a few percent.

There are a few available literature data to compare our results with. The results of numerical simulations reported by Marschall and Teitel [33] for rounded crosses with aspect ratio of $\rho=0.5$ is in excellent agreement with our experimental findings of $0.62<\phi<0.66$ for the rounded crosses with $\rho=0.445$ and $0.67<\phi<0.71$ for rounded crosses with $\rho=0.53$.

These pair correlation functions reported by these authors also appear very similar to our findings in Fig. 12. Marschall and Teitel presented the numerically computed orientational correlations not as a function of the cross separation $r$, but they distinguished between neighbors in the directions along the arms and in the directions $45^{\circ}$ respective to the arms. As in our experiments, the density correlations vanish at distances larger than $\approx 3 \ldots 4 r_{0}$. The authors also computed the tetratic correlation, also separately for neighboring crosses in the direction along the arms and diagonal to them. The features of these plots are qualitatively very similar to our experimental data. A first positive peak $Q_{4}\left(r_{0}\right) \approx 1$ is 
followed by a negative peak and thereafter alternating excursions of $Q_{4}$ to positive and negative peaks until the correlations vanish at around 3 to $4 r_{0}$.

The stress-birefringent crosses studied by Zheng et al. [31], practically equivalent to rounded crosses with $\rho=0.4$, show a jamming transition after uniaxial compression at $\phi \approx 0.475 \pm 0.002$. Interestingly, this value is considerably smaller than in our experiments with similar crosses, and also much smaller than the value reported by Marschall and Teitel [33] for comparable objects. The reason could be a considerably higher friction coefficient ( 0.7$)$ of these crosses. This observation might be a hint that a systematic study of the influence of the friction coefficient on the jamming transition of crosses is desirable.

Another interesting complexion of this problem is the introduction of restricted geometries. Our experiment already shows (Fig. 5) that orientational order is induced by the boundaries, and thus the restriction to ensembles of crosses to narrow channels may lead to structural transitions when the gap width is gradually changed. A similar scenario has been investigated by Gurin et al. [48] and Bautista-Carbajal et al. [35] for squares (crosses in the limit $\rho \rightarrow \infty$ ) in a thin slit. Depending upon the width $H$ of the slit relative to the side length of the squares, the maximum packing fractions of the structures were determined. They vary between 0.55 and 1, undergoing several structural transitions with increasing $H$. Coexisting structures are observed near the transition points.

Summarizing, we have proposed regular (crystalline) structures that provide the highest packing fractions of crosses over the full parameter range of aspect ratios from $\rho=0$ (crossed line segments) to $\infty$ (squares). Depending upon $\rho$, four qualitatively different structures have been identified that presumably provide the best packing. The results were obtained for crosses with flat ends. For crosses with rounded arm ends [32], the optimal structures apparently provide lower packing fractions, at least for $\rho<1$.

In experiments, we have determined the jamming transitions of crosses with aspect ratios between 0.25 and 2 . The packing structures achieved by uniaxial compression are satisfactorily isotropic in the bulk, while at the moving edge some alignment is induced. This alignment leads to a negative tetratic order parameter at the boundary (crosses diagonal to the edge) when the aspect ratio is small, and to a slightly positive tetratic order parameter (arms along and perpendicular to the edge) at large $\rho$. Our estimate is that the transition between the two alignment scenarios occurs at $\rho_{c}=\sqrt{1 / 2}$.

The orientational correlation parameter $Q_{4}$ in the jammed configurations has been determined from the experimental images. While crosses in direct contact $\left(r=r_{0}\right)$ create a peak at perfect order $Q_{4}\left(r_{0}\right)=1$, this peak is followed by a dip of negative $Q_{4}$ and several oscillations of $Q(r)$. These decay over a range of about $3 \ldots 4 r_{0}$, this is about the size of 2 crosses. Over larger distances, the correlations are lost.

Positional correlations $\phi_{\text {loc }}(r)$ were also determined. We observe several local peaks which can be attributed to special arrangements of groups of crosses. The correlations decay over distances of about $3 \ldots 4 r_{0}$, like the orientational correlations. The average packing fractions in the experiments have been determined as a function of $\rho$. The general trend is that we achieve roughly $70 \%$ of the maximum packing fractions. Rounded crosses show a slight tendency to pack tighter.

The results of this study may serve as a guide to select parameters for further experiments with cross-shaped particles such as shear experiments or discharge of non-convex particles from containers with narrow orifices (e.g. [49]). It may also help to judge the accuracy of simulations and provide a basis for an estimation of the stability of packing structures.

\section{Appendix A: Optimum crystalline packings}

We recollect the regular crystalline arrangements found to be the best space-filling lattices, described earlier in the Supplemental Material of Ref. [34]. Atkinson's study considers crosses with straight edges. While we are not aware of a strict mathematical proof that all the structures described in the following are indeed the optimal arrangements, they definitely represent lower limits for the optimum packing fraction $\phi_{\max }$. The corresponding packing structures for flatended crosses (Fig. 1 right) are shown in Fig. 2.

The densest crystalline packing at small aspect ratios $\rho=b / a$ is the tilted square, TSQ: Two arms of adjacent crosses lay next to each other side by side. The unit cell is indicated by a dashed square in the Figure. It is tilted respective to the arms of the cross by an angle $\beta=\arctan [b /(a+b)]=\arctan [\rho /(1+\rho)]$. There is one cross per unit cell, as in all other configurations described in this section. The length of the vectors spanning the unit cell is $r_{\mathrm{T}}=\sqrt{(a+b)^{2}+b^{2}}$. Since the area of a single cross is $A_{0}=4 a b+b^{2}=a^{2}\left(4 \rho+\rho^{2}\right)$, and the area of the unit cell is $A_{\mathrm{T}}=(a+b)^{2}+b^{2}=a^{2}\left[(1+\rho)^{2}+\rho^{2}\right]$. The packing fraction is

$\phi_{(\mathrm{TSQ})}=\frac{A_{0}}{A_{\mathrm{T}}}=\frac{4 \rho+\rho^{2}}{(1+\rho)^{2}+\rho^{2}}$.

It reaches its maximum value one for an aspect ratio $\rho=1$ (see Fig. 3). When $\rho$ increases further, it decays again. The graph of $\phi_{(\mathrm{TSO})}$ is shown in Fig. 3a as a black solid curve in the range $\rho<(1+\sqrt{5}) / 2$ where it provides the best area filling, and as a blue dashed line for larger $\rho$ where other 
arrangements are superior. It reaches the asymptotic value $1 / 2$ for $\rho \rightarrow \infty$.

At $\rho>(1+\sqrt{5}) / 2 \approx 1.618$, the next candidate for optimal packing is the rhombic unit cell (shown in Fig. 2 for $\rho=1.5$ ). We label it ETE (end to end), since two crosses touch each other with the ends of their arms, e. g. the green and orange one in the figure. The cyan and yellow ones touch the arms of these crosses from the sides. The unit cell area is equal to that of the rhombus drawn in Fig. 2, with side lengths $a+b$ and $2 a+b$, the area is $A_{\mathrm{E}}=a^{2}\left[(1+\rho)^{2}+1+\rho\right]$, and the corresponding fill fraction

$\phi_{(\mathrm{ETE})}=\frac{A_{0}}{A_{\mathrm{E}}}=\frac{4 \rho+\rho^{2}}{(1+\rho)^{2}+1+\rho}$.

For low $\rho$, this structure has a lower fill fraction than the tilted square configuration described above, but at fill fractions $(1+\sqrt{5}) / 2<\rho<2$ the packing is more efficient than in all competing configurations. This ETE structure exists up to $\rho=2$ where again, the perfect tiling $\phi=1.0$ is achieved. For $\rho>2$, another structure with a rhombic unit cell is found which is more efficient than the tilted square TSQ. Crosses are stacked with arms side by side (SBS) alternating with their upper and lower arms, as seen in the $\rho=2.5$ example in Fig. 2. The unit cell is again a rhombus, as shown in the figure, but one can also choose a parallelogram with a side length of $2 b$ and a height $a+b$. The unit cell area is $A_{\mathrm{S}}=2\left(\rho+\rho^{2}\right) a^{2}$. The packing fraction becomes

$\phi_{(\mathrm{SBS})}=\frac{A_{0}}{A_{\mathrm{S}}}=\frac{4 \rho+\rho^{2}}{2 \rho+2 \rho^{2}}$.

The ETE structure has one peculiarity that distinguishes it from the previous lattice. It consists of loosely coupled sublattices and it has a soft mode: The two sublattices of crosses stacked end-to-end in a line can be shifted with respect to each other by displacements $\pm(a-b / 2)= \pm(1-\rho / 2) a$, while the global packing fraction remains unchanged.

Finally, for $\rho>1+\sqrt{3} \approx 2.732$, another structure provides the optimum packing, which we will refer to as tilted rhomboid, TRH. The vectors spanning the unit cell have lengths of $\sqrt{(2 a+b)^{2}+4 a^{2}}$ and $\sqrt{(a+b)^{2}+b^{2}}$, The packing fraction is

$\phi_{(\mathrm{TRH})}=\frac{A_{0}}{A_{\mathrm{TRH}}}=\frac{4 \rho+\rho^{2}}{\rho^{2}+4 \rho+2}$.

At $\rho \rightarrow \infty(a=0)$, the crosses degenerate to squares, and the packing fraction reaches $\phi=1$ for the third time. The complete graph of the fill fractions corresponding to the regular structures defined above is shown in Fig. 3a. The dashed lines show the fill fractions of the different configurations where they exist but are no optimum structures. The solid black line is the plot of the optimum configurations for given $\rho$.

Several other regular configurations with more than one cross per unit cell are shown in Appendix B. None of them reaches a higher packing fraction than the optimum configurations described above.

We emphasize that we have only considered regular packing configurations. Even though there is great confidence that the most efficient packing structures have been identified [34], we have no mathematical proof that denser configurations cannot exist. The main idea of introducing these lower limits of densest packing here was to set a scale for the evaluation of the experimental results on randomly packed crosses. This is described in the following sections.

\section{Appendix B: Other crystalline configurations}

In addition to the optimum lattices shown in Appendix A, it is worthwhile to mention a candidate that does not reach the optimum packing fraction but is nevertheless interesting in non-perfect lattices. It can be described by an nontilted square unit cell, and we denote it by SQ. The side length of the unit cell is $r_{\mathrm{SQ}}=\sqrt{(2+\rho)^{2} a^{2}}$, the area is $A_{\mathrm{Sq}}=(2+\rho)^{2} a^{2}$. The structure is exemplarily shown for $\rho=4$ in Fig. 15. It has a packing fraction of

$\phi_{(\mathrm{SQ})}=\frac{A_{0}}{A_{\mathrm{SQ}}}=\frac{4 \rho+\rho^{2}}{(2+\rho)^{2}}$.

(green dashes in Fig. 3).

This structure is interesting because, unlike TRH, this one is infinitely degenerate. One can shift either individual rows or individual columns arbitrarily, keeping $\phi$ unchanged. This structure can be considered a smectic arrangement, with layers that can be shifted respective to their neighbors. For the optimum space filling, this structure is less suited than TRH

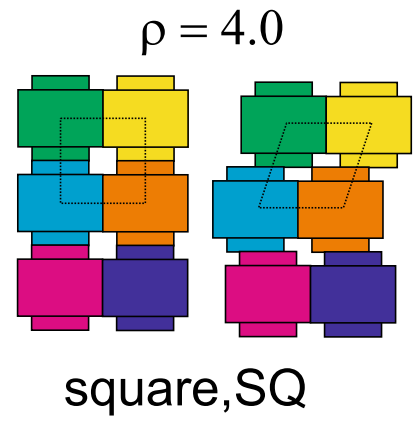

Fig. 15 A configuration which is not optimal for any aspect ratios, but may compete with TRH in systems that can explore the configuration space (by external excitation or thermal motion), because of its large entropy (positional degeneracy) 
(a)

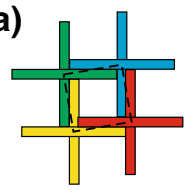

$\downarrow \rho=0.2$

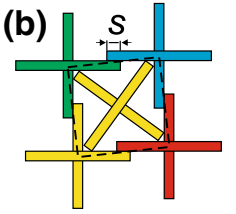

FTSQ (c)

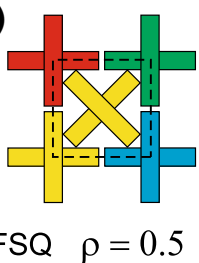

(d)

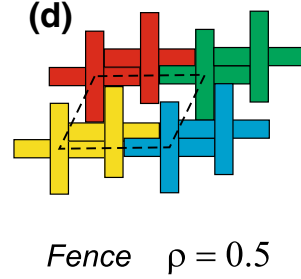

(e)

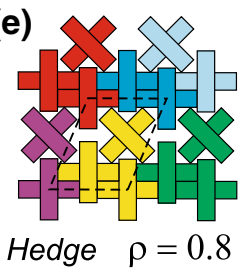

(f)

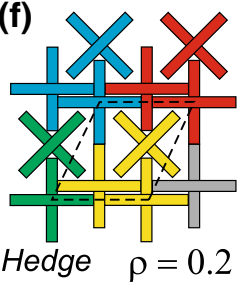

(g)

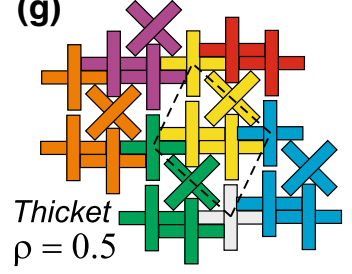

(h)

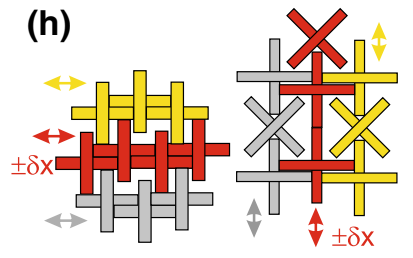

Fig. 16 Configurations with more than one cross per unit cell (indicated by dashed lines). a, b Transformation of the TSQ into a FTSQ lattice; c filled square; $\mathbf{d}$ the Fence with two crosses per_rhomboid unit cell; e the Hedge with three wide crosses $(\rho>2-\sqrt{2})$ per unit cell; f same with thin crosses $(\rho<2-\sqrt{2}$; g more complex struc-

at all aspect ratios, but if one considers systems with thermal motion, it may be entropically advantageous.

So far, our analysis was restricted to configurations with one cross per unit cell. In order to probe into other regular lattices, For very small $\rho$, one might assume that a slight modification of the TSQ arrangement is more efficient. The unit cell is enlarged and filled, viz. a diagonal cross is inserted in the center (Figs. 16 a,b). This creates the filled tilted square structure, FTSQ. The optimum angle $\gamma$ of the central cross respective to the four outer crosses is related to the aspect ratio by $\sin 2 \gamma=1-\rho^{2}$. The outer crosses have to be shifted outward so that the arm lengths do not overlap along their full width $a$, but only by a length $s=2 a-(b+a) \sqrt{2-b^{2} / a^{2}}$ (Fig. 2). Therefore, the unit cell has edge lengths $r_{\mathrm{FT}}=a \sqrt{\left(\rho+(1+\rho) \sqrt{2-\rho^{2}}\right)^{2}+\rho^{2}}$. Now, there are two crosses per FTSQ unit cell. It turns out that the resulting fill fraction

$\phi_{(\mathrm{FTSQ})}=\frac{2 A_{0}}{A_{\mathrm{FT}}}=\frac{8 \rho+2 \rho^{2}}{\left(\rho+(1+\rho) \sqrt{2-\rho^{2}}\right)^{2}+\rho^{2}}$

is generally lower than that of TSQ for all aspect ratios $\rho>0$. We can discard this configuration as candidate for the optimum packing. At $\rho \approx 0.52$, the overlap $s$ reaches zero and the configuration does not exist further. The graph of this function is shown in Fig. 17.

The inner cross can also be inserted symmetrically when the arms of the outer frame do not overlap, as shown in Fig. 16c). The area of the quadratic unit cell of this Filled Square (FSQ) is $A_{\mathrm{FS}}=[\sqrt{(2)}(a+b)+b]^{2}$, and the packing fraction, with two crosses per unit cell, is ture ('Thicket') with four crosses per unit cell. $\mathbf{h}$ demonstrates that the Fence and the thin cross Hedge in (d) and (f) are infinitely degenerate. They consist of sub-lattices (here: equally colored crosses in subfigure h) that can be independently shifted relative to their neighbors by small displacements $\delta x$

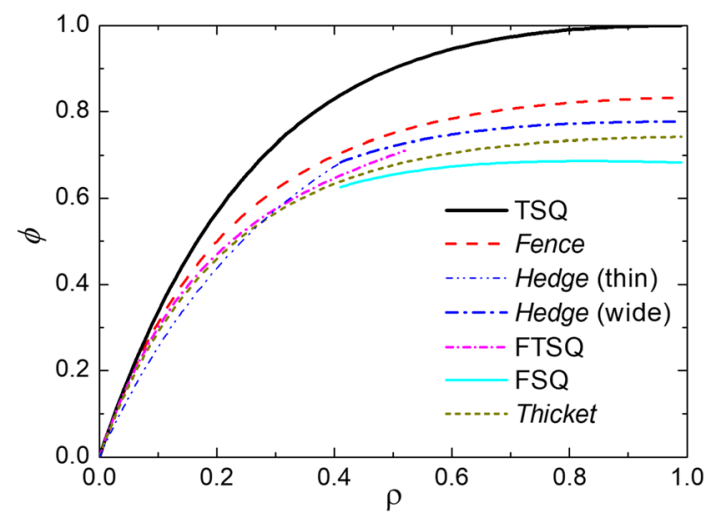

Fig. 17 Packing fractions of selected configurations in the range $\rho<1$. The corresponding unit cells are shown in Fig. 16. Some of the configurations do not exist in the complete parameter range. For example, there is a crossover between the Hedge structures at $\rho=2-\sqrt{(} 2)$

$\phi_{(\mathrm{FSQ})}=\frac{2 A_{0}}{A_{\mathrm{FS}}}=\frac{8 \rho+2 \rho^{2}}{(\sqrt{2}(1+\rho)+\rho)^{2}}$

It is less efficient than FTSQ everywhere. For $\rho<\sqrt{2}-1$ in particular, the outer crosses cannot get closer since their arms forming the sides of the unit cell touch each other. Then, its packing efficiency is even worse than predicted by an extrapolation of Eq. (10).

Another promising configuration, with all crosses in the same orientation, is the 'Fence', shown in Fig. 16d. The rhomboid unit cell with area $A_{\mathrm{Fe}}=[(2 a+2 b)(a+2 b)]^{2}$ is sketched by dashed lines. It contains two crosses per unit cell, and 
$\phi_{(\text {Fence })}=\frac{2 A_{0}}{A_{\mathrm{Fe}}}=\frac{8 \rho+2 \rho^{2}}{(2+2 \rho)^{2}(1+2 \rho)^{2}}$.

As is seen in Fig. 17, it indeed provides the best packing fraction of all considered structures except TSQ.

Three crosses per unit cell are contained in the structures in Fig. 16e,f, the 'Hedge'. There are two different configurations: For thin crosses $(\rho<2-\sqrt{2})$, the diagonal cross touches the end of the vertical arms of the upper and lower neighbors, and for wide crosses, it touches the horizontal arms of the upper and lower crosses at their sides. The unit cell areas are $A_{\mathrm{Hw}}=(2 a+2 b)(\sqrt{2}(a+b)+2 b)$ for the wide crosses $(\rho>2-\sqrt{2})$ and $A_{\mathrm{Ht}}=(2 a+2 b)^{2}$ for the thin crosses $(\rho<2-\sqrt{2})$. Packing fractions are shown as blue lines in Fig. 17. One may conceive other crystalline configurations, even such with more than three crosses per unit cell (e. g. four in the 'Thicket', Fig. 16g). They all fail to provide a better packing than the optimum, TSQ. For the Thicket shown in the image, for example, one finds a packing fraction

$\phi_{(\text {Thicket })}=\frac{4 A_{0}}{A_{\mathrm{Th}}}=\frac{16 \rho+4 \rho^{2}}{(1+\rho)(3 \sqrt{2}(1+\rho)+5 \rho)}$.

It is comparable to those of the FTSQ and Hedge structures. This list is by far not complete. Generally, it seems that packing diagonal crosses into unit cells brings no advantages over structures where all crosses are oriented uniformly.

In addition to the positional ordering, the configurations shown in Fig. 16 differ in their orientational order parameters. The tetratic order parameter $q_{4}$ of FSQ and FTSQ is zero, for the Hedge it is $1 / 3$, for the Thicket $1 / 4$, and the fence has $q_{4}=1$.

Finally, we note a certain peculiarity of two of the structures, demonstrated in Fig. 16h: The Fence and the thin Hedge both possess a soft mode, they consist of one-dimensional sub-lattices. Horizontal rows of the Fence in Fig. 16d can be shifted respective to their neighbor rows by a distance $\delta x$ of at most $a(1-\rho)$ without changing the packing fraction. The structure is therefore infinitely degenerate. The same applies to the Hedge of Fig. 16f. Each vertical line can be shifted with respect to its neighbors by a small displacements $|\delta x|<(2-\sqrt{2}(1+\rho)) a$.

\section{Appendix C: Surface induced order}

When a cross gets in contact with the moving barrier, a torque is generated by the force of the moving barrier acting at the contact point and the inertia force acting on the center of mass. Depending on the sign of this torque, the cross has two possible reorientations, either towards $\alpha_{i}=0$ or towards $\alpha_{i}=\pi / 4$ (Fig. 18). The critical angle separating these two cases is $\alpha_{c}=\pi / 2-\arctan (2 \rho+1)$. The probabilities are

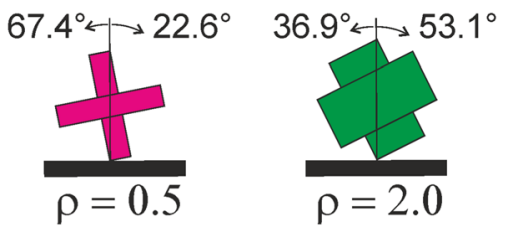

Fig. 18 Crosses in contact with the wall at one corner: Depending on the angle between cross and barrier, the cross rotates either towards $\alpha_{i}=\pi / 4$ (here counterclockwise) or $\alpha_{i}=0$ (here clockwise). The probabilities relate to angular ranges $2 \alpha_{c}$ and $\pi / 2-2 \alpha_{c}$ corresponding to the two scenarios. Their widths are given for the two selected $\rho$

Table 2 Minimal number of crosses used in each experiment and number of repetitions of the experiments. In each individual experiment, three images are recorded

\begin{tabular}{llll}
\hline Nominal & Aspect ratio $\rho$ & No. of crosses & No. of exp. \\
\hline $3 \mathrm{~mm}$ & 0.248 & 401 & 100 \\
$2 \mathrm{~mm}$ & 0.284 & 500 & 100 \\
$4 \mathrm{~mm}$ & 0.445 & 400 & 50 \\
$5 \mathrm{~mm}$ & 0.516 & 400 & 100 \\
$8 \mathrm{~mm}$ & 0.728 & 312 & 100 \\
$10 \mathrm{~mm}$ & 0.894 & 191 & 100 \\
black & 2.0 & 166 & 50 \\
\hline
\end{tabular}

equal for $\alpha_{c}=\pi / 8(\rho=\sqrt{1 / 2})$. Thicker crosses prefer alignment parallel to the walls, thinner ones prefer diagonal surface alignment.

\section{Appendix D: Experimental parameters}

Table 2 lists the number of experiments and the number of crosses for each type of crosses.

Acknowledgements The authors kindly acknowledge Torsten Trittel and Christoph Klopp for 3D-printing crosses, and Eckard Specht for stimulating discussions. The German Science Foundation is acknowledged for funding within project STA 425/46-1.

Funding Open Access funding enabled and organized by Projekt DEAL.

Open Access This article is licensed under a Creative Commons Attribution 4.0 International License, which permits use, sharing, adaptation, distribution and reproduction in any medium or format, as long as you give appropriate credit to the original author(s) and the source, provide a link to the Creative Commons licence, and indicate if changes were made. The images or other third party material in this article are included in the article's Creative Commons licence, unless indicated otherwise in a credit line to the material. If material is not included in the article's Creative Commons licence and your intended use is not permitted by statutory regulation or exceeds the permitted use, you will 
need to obtain permission directly from the copyright holder. To view a copy of this licence, visit http://creativecommons.org/licenses/by/4.0/.

\section{References}

1. Hales, T.C.: A proof of the Kepler conjecture. Ann. Math. 162, 1065-1185 (2005)

2. McGeary, R.K.: Mechanical packing of spherical particles. J. Am. Ceram. Soc. 44, 513 (1961)

3. Mughal, A., Chan, H.K., Weaire, D., Hutzler, S.: Dense packings of spheres in cylinders: simulations. Phys. Rev. E 85, 051305 (2012)

4. Reimann, J., Abou-Sena, A., Nippen, R., Tafforeau, P.: Pebble bed packing in prismatic containers. Fusion Eng. Des. 88, 2343 (2013)

5. Harth, K., Mauney, A., Stannarius, R.: Frustrated packing of spheres in a flat container under symmetry-breaking bias. Phys. Rev. E 91, 030201(R) (2015)

6. Lévay, S., Fischer, D., Stannarius, R., Szabó, B., Börzsönyi, T., Török, J.: Frustrated packing in a granular system under geometrical confinement. Soft Matter 14, 396 (2018)

7. Donev, A., Cisse, I., Sachs, D., Variano, E., Stillinger, F., Connelly, R., Torquato, S., Chaikin, P.: Improving the density of jammed disordered packings using ellipsoids. Science 303, 990 (2004)

8. Torquato, S., Jiao, Y.: Dense packings of polyhedra: platonic and Archimedean solids. Phys. Rev. E 80, 041104 (2009)

9. Haji-Akbari, A., Engel, M., Keys, A.S., Zheng, X., Petschek, R.G., Palffy-Muhoray, P., Glotzer SCl,: Disordered, quasicrystalline and crystalline phases of densely packed tetrahedra. Nature 462, 773 (2009)

10. Damasceno, P.F., Engel, M., Glotzer, S.C.: Predictive self-assembly of polyhedra into complex structures. Science 337, 453 (2012)

11. Haji-Akbari, A., Chen, E.R., Engel, M., Glotzer, S.C.: Packing and self-assembly of truncated triangular bipyramids. Phys. Rev. E 88, 012127 (2013)

12. Teich, E.G., van Anders, G., Klotsa, D., Dshemuchadse, J., Glotzer, S.C.: Clusters of polyhedra in spherical confinement. PNAS 113, E669 (2016)

13. Kyrylyuk, A.V., van de Haar, M.A., Rossi, L., Wouterse, A., Philipse, A.P.: Isochoric ideality in jammed random packings of non-spherical granular matter. Soft Matter 7, 1671-1674 (2011)

14. Zhao, Y., Liu, K., Zheng, M., Barés, J., Dierichs, K., Menges, A., Behringer, R.P.: Packings of 3D stars: stability and structure. Granular Matter 18, 1 (2016)

15. Zhao, Y., Barés, J., Socolar, J.E.S.: Yielding, rigidity, and tensile stress in sheared columns of hexapod granules. Phys. Rev. E 101, 062903 (2020)

16. Qi, W., de Graaf, J., Qiao, F., Marras, S., Manna, L., Dijkstra, M.: Ordered two-dimensional superstructures of colloidal octapodshaped nanocrystals on flat substrates. Nano Lett. 12, 5299 (2012)

17. Dierichs, K., Menges, A.: Granular construction Architectural Design 8586 (2015); towards an aggregate architecture: designed granular systems as programmable matter in architecture. Granular Matter 18, 25 (2016)

18. Keller, S., Jaeger, H.: Aleatory architectures. Granular Matter 18, 29 (2016)

19. Torquato, S., Stillinger, F.H.: Jammed hard-particle packings: from Kepler to Bernal and beyond. Rev. Mod. Phys. 82, 2633 (2010)

20. Radin, C.: Random close packing of granular matter. J. Stat. Phys. 131, 567 (2008)
21. Knight, J.B., Fandrich, C.G., Lau, C.N., Jaeger, H.M., Nagel, S.R.: Density relaxation in a vibrated granular material. Phys. Rev. E 51, 3957 (1995)

22. Clément, E., Labous, L., Vanel, L.: Granular packing under vibration. Europhys. News 29, 107-111 (1998)

23. Richard, P., Nicodemi, M., Delannay, R., Ribiere, P., Bideau, D.: Slow relaxation and compaction of granular systems. Nat. Mater. 4, 121 (2005)

24. Anikeenko, A.V., Medvedev, N.N.: Polytetrahedral nature of the dense disordered packings of hard spheres. Phys. Rev. Lett. 98, 235504 (2007)

25. Kapfer, S. C., Mickel, W., Mecke, K., Schröder-Turk, G. E.: Jammed spheres: Minkowski tensors reveal onset of local crystallinity. Phys. Rev. E 85030301 (R) (2012)

26. Rietz, F., Radin, C., Swinney, H.L., Schröter, M.: Nucleation in sheared granular matter. Phys. Rev. Lett. 120, 055701 (2018)

27. Fejes Tóth, L.: Lagerungen in der Ebene, auf der Kugel und im Raum, Die Grundlehren der mathematischen Wissenschaften. 65, Springer, Berlin (1972)

28. Xu, Y., Barés, J., Zhao, Y., Behringer, R.P.: Jamming transition: heptagons, pentagons, and discs. EPJ Web Conf. 140, 06010 (2017)

29. Zhao, Y., Barés, J., Zheng, H., Bester, C. S., Xu, Y., Socolar, J. E. S., Behringer, R. P.: Jamming transition in non-spherical particle systems: pentagons versus disks. Granular Matter 2190 (2019)

30. Schreck, C., Xu, N., O'Hern, C.S.: A comparison of jamming behavior in systems composed of dimer- and ellipse-shaped particles. Soft Matter 6, 2960 (2010)

31. Zheng, H., Wang, D., Barés, J., Behringer, R.: Jamming by compressing a system of granular crosses. EPJ Web Conf. 40, 06014 (2017)

32. Meng, L., Yao, Xiaohu., Zhang, X.: Two-dimensional densely ordered packings of non-convex bending and assembled rods. Particuology 5035 (2020)

33. Marschall, T.A., Teitel, S.: Athermal shearing of frictionless cross-shaped particles of varying aspect ratio. Granular Matter 22, 4 (2020)

34. Atkinson, S., Jiao, Y., Torquato, S.: Maximally dense packings of two-dimensional convex and concave noncircular particles. Phys. Rev. E 86, 031302 (2012)

35. Bautista-Carbajal, G., Gurin, P., Varga, S., Odriozola, G.: Phase diagram of hard squares in slit confinement. Sci. Rep. 8, 8886 (2018)

36. Macrae, J.C., Finlayson, P.C., Gray, W.A.: Vibration packing of dry granular solids. Nature 179, 1365 (1957)

37. Nowak, E.R., Knight, J.B., Povinellli, M.L., Jaeger, H.M., Nagel, S.R.: Reversibility and irreversibility in the packing of vibrated granular material. Powder Technol. 94, 79 (1997)

38. Rémond, S., Gallias, J.L.: Simulation of periodic mono-sized hard sphere systems under different vibration conditions and resulting compaction. Phys. A 369, 545 (2006)

39. Rosato, A.D., Dybenko, O., Horntrop, D.J., Ratnaswamy, V.: and Lou Kondic, Microstructure evolution in density relaxation by tapping. Phys. Rev. E 81, 061301 (2010)

40. Sánchez, R., Aguirre-Manzo, L.A.: Concentric tetratic orientational order in a confined quasi-2D tubular system. Phys. Scr. 90, 095002 (2015)

41. Walsh, L., Menon, N.: Ordering and Dynamics of Vibrated Hard Squares. J. Stat. Mech. 083302 (2016)

42. Windows-Yule, C.R.K.: Do granular systems obey statistical mechanics? A review of recent work assessing the applicability of equilibrium theory to vibrationally excited granular media. Int. J. Mod. Phys. B 31, 1742010 (2017)

43. Donev, A., Burton, J., Stillinger, F.H., Torquato, S.: Tetratic order in the phase behavior of a hard-rectangle system. Phys. Rev. B 73, 054109 (2006) 
44. Wojciechowski, K.W., Frenkel, D.: Tetratic phase in the planar hard square system? Comput. Met. Sci. Technol. 10, 235 (2004)

45. Martínez-González, J.A., Armas-Pérez, J.C., Quintana, H.J.: Phase behavior of bow-shaped hard particles in two dimensions. J. Stat. Phys. 150, 559 (2013)

46. Cadotte, A.T., Dshemuchadse, J., Damasceno, P.F., Newman, R.S., Glotzer, S.C.: Self-assembly of a space-tessellating structure in the binary system of hard tetrahedra and octahedra. Soft Matter 12, 7073 (2016)

47. Hou, Z., Zong, Y., Sun, Z., Ye, F., Mason, T.G., Zhao, K.: Emergent tetratic order in crowded systems of rotationally asymmetric hard kite particles. Nat. Commun. 11, 2064 (2020)
48. Gurin, P.S., Odriozola, G.: Anomalous structural transition of confined hard squares. Phys. Rev. E 94 050603(R) (2016)

49. Hafez, A., Liu, Q., Finkbeiner, T., Alouhali, R.A., Moellendick, T.E., Santamarina, J.C.: The effect of particle shape on discharge and clogging. Sci. Rep. 11, 3309 (2021)

Publisher's Note Springer Nature remains neutral with regard to jurisdictional claims in published maps and institutional affiliations. 